MATHEMATICS OF COMPUTATION

Volume 70, Number 233, Pages 1-15

S $0025-5718(00) 01323-5$

Article electronically published on October 2, 2000

\title{
SHAPE CALCULUS AND FINITE ELEMENT METHOD IN SMOOTH DOMAINS
}

\author{
T. TIIHONEN
}

\begin{abstract}
The use of finite elements in smooth domains leads naturally to polyhedral or piecewise polynomial approximations of the boundary. Hence the approximation error consists of two parts: the geometric part and the finite element part. We propose to exploit this decomposition in the error analysis by introducing an auxiliary problem defined in a polygonal domain approximating the original smooth domain. The finite element part of the error can be treated in the standard way. To estimate the geometric part of the error, we need quantitative estimates related to perturbation of the geometry. We derive such estimates using the techniques developed for shape sensitivity analysis.
\end{abstract}

In this paper we consider the dilemma of "smooth polygonal domains" related to error analysis of the finite element method. The dilemma is that the finite element methods are naturally formulated in polygonal (or more generally in piecewise polynomial) geometries. On the other hand, the abstract error estimates rely on interpolation error estimates that require smoothness of the solution that is typically achieved only in regular geometries.

In the literature this question has been treated in several ways. Perhaps the most popular approach is that of the above mentioned smooth polygonal domains. That is, the analysis is carried out assuming that the domain is polygonal and hence the grid fits exactly to the domain, and at the same time the solution is regular enough for the optimal interpolation estimates.

At the other extreme are the works where the curved boundary is captured more or less exactly by introducing corresponding curved elements, 4], 5], 7], 16]. In this case the analysis can be made rigorous but the price to pay is more complicated local analysis and implementation, especially if the basis functions are modified in the curved elements. If one does not want to modify the basis functions near the boundary, then the approach of [3] provides a way to analyse the resulting variational crime on the boundary. In [7] this approach has been used to construct optimal interpolated essential boundary conditions for curved boundaries.

Finally, there exists quite a number of papers where the smooth domain is approximated by a polygonal one which is then triangulated. In many of the papers the error analysis is based on some specific feature, like trivial extension of the finite element solution outside of the polygonal domain. Also the analysis of the

Received by the editor November 17, 1997.

2000 Mathematics Subject Classification. Primary 65N30; Secondary 49Q12.

Key words and phrases. Finite elements, curved boundary, error estimates, shape derivatives, continuous dependence on geometry. 
approximation of geometry is generally interwoven with the analysis of the finite element approximation properties.

In this paper we introduce an approach where the approximation of geometry is detached from finite element analysis. This means that we shall analyze the error made due to replacing the original problem in a smooth domain by an auxiliary problem in a (polygonal) approximate domain. Then we analyse the error for the finite element approximation of the auxiliary problem, bearing in mind that the auxiliary solution is close to the original smooth one. The first error is estimated using the techniques familiar from shape optimization [9] where the question of continuous dependence with respect to variations in geometry is a key issue.

The question of continuous dependence of the solution on the geometric data dates back to at least Hadamard's times. Most of the analysis has been qualitative. Some quantitative works were published in early 1970's ([10], [11]) with the motivation arising from finite element error estimates. Then, it seems, the issue was forgotten.

The contents of this paper can be summarized briefly as follows. In Section 1 we discuss the strategy of decomposing the error to geometric and finite element components in an abstract framework. Then in Section 2 we consider a model second order elliptic problem for which we show the continuous dependence of the solution under polyhedral approximation of the boundary. Corresponding finite element error estimates are also formulated. In subsequent sections we consider second order systems and fourth order problems where polyhedral approximation of the boundary turns out to be a much more delicate issue. In particular we comment on Babuska's famous counterexamples ([1], [2]) from the point of view of shape derivatives.

\section{Abstract Formulation}

The aim is to study the dependence between the solution $u$ of the variational problem defined in a smooth domain $\Omega$ and its finite element approximation $u_{h}$ defined in an approximate domain $\Omega_{h}$. To be able to compare $u$ and $u_{h}$, we have to be able to prolongate one of the two to the domain of definition of the other. As prolongation of finite element functions can be technically difficult (as finite element functions) in the general case, we choose to prolongate the original solution from $\Omega$ to $\Omega_{h}$ by some prolongation operator. The idea behind error estimation is to introduce an auxiliary problem defined in $\Omega_{h}$ to be able to separate the approximation of geometry from approximation by finite element spaces.

Thus, let the original problem be given as

$$
a(u, w)=\langle f, w\rangle \quad \forall w \in W
$$

for $f \in W^{\prime}, u \in W=W(\Omega)$. We introduce an auxiliary problem

$$
\hat{a}(\hat{u}, w)=\langle\hat{f}, w\rangle \quad \forall w \in \hat{W}
$$

with $\hat{W}=\hat{W}(\hat{\Omega})$. The bilinear forms $a$ and $\hat{a}$ are assumed to be continuous and $W$ (resp. $\hat{W}$ ) elliptic. The auxiliary problem is defined so that its finite element discretization gives the discrete problem

$$
\hat{a}\left(u_{h}, w_{h}\right)=\left\langle\hat{f}, w_{h}\right\rangle \quad \forall w_{h} \in W_{h} \subset \hat{W} .
$$

We now want to estimate the error between $u$ and $u_{h}$. Because of different domains of definition we have to consider an extension $\tilde{u}$ of $u$. Let $\|\cdot\|$ be some 
norm for functions defined in $\Omega_{h}$. Then, by the triangle inequality,

$$
\left\|\tilde{u}-u_{h}\right\| \leq\|\tilde{u}-\hat{u}\|+\left\|\hat{u}-u_{h}\right\| .
$$

Here the first part corresponds to the error due to geometry, whereas the second part is a standard finite element approximation error (but now in a polygonal domain).

Assume now that we have a quasi-optimality result with respect to the $\|\cdot\|$ norm. That is,

$$
\left\|\hat{u}-u_{h}\right\| \leq C \inf _{w_{h} \in W_{h}}\left\|\hat{u}-w_{h}\right\| .
$$

Then, using the triangle inequality, again we can estimate

$$
\left\|\hat{u}-w_{h}\right\| \leq\|\hat{u}-\tilde{u}\|+\left\|\tilde{u}-w_{h}\right\| .
$$

Hence,

$$
\left\|\tilde{u}-u_{h}\right\| \leq C_{1}\|\tilde{u}-\hat{u}\|+C_{2} \inf _{w_{h} \in W_{h}}\left\|\tilde{u}-w_{h}\right\| .
$$

In order to derive useful concrete error estimates from (1.2), we have to make sure that the following conditions are satisfied.

1. $\hat{f}, \hat{a}$ and the extension operator are chosen so that we get an appropriate estimate for $\|\tilde{u}-\hat{u}\|$.

2. Extension is defined so that $\tilde{u}$ has the regularity needed for interpolation estimates, that is, $\tilde{u}$ should be smooth when restricted to any element of the triangulation.

3. $\hat{f}$ and $\hat{a}$ should be natural extensions of $f$ and $a$ as they will be used to construct the discrete problem.

4. The quasi-optimality estimate (1.1) is valid uniformly for all $\Omega_{h}$.

Condition 1 above is best satisfied when the auxiliary problem is defined simply by mapping $\Omega$ to $\hat{\Omega}$ and defining the "extensions" using the same transformation. Then, of course $\|\tilde{u}-\hat{u}\|=0$. On the other hand the mapping shows up in the coefficients of the auxiliary problem and its discretization complicating thus both error analysis and implementation. This is what happens in practice with curved elements.

Condition 4 is satisfied at least for the energy norm for $W$-elliptic problems.

Finally, let us remark that the above abstract estimate can be extended, for example, to cases where we compare the solution to the post-processed discrete solution $R u$, where $R$ is some recovery operator, provided that we have an estimate of the type

$$
\left\|\hat{u}-R u_{h}\right\| \leq C \inf _{w_{h} \in W_{h}}\left\|\hat{u}-R w_{h}\right\|
$$

that is valid uniformly in $\Omega_{h}$ :s. Likewise, the quasi-optimality estimate (1.1) can be replaced by a more general one to cope with numerical quadrature, for example.

\section{Model PROBlem}

Let us consider the following model problem:

$$
\begin{aligned}
-\Delta u & =f, & & \text { in } \Omega, \\
u & =u_{0}, & & \text { on } \Gamma_{D}, \\
\frac{\partial u}{\partial n} & =g, & & \text { on } \Gamma_{N}
\end{aligned}
$$


where $\Omega \subset D \subset R^{n}$ is a smooth domain. For our convenience we assume that the complement of $\Omega$ is not connected, and the boundary $\partial \Omega$ can decomposed to $\Gamma_{D}$ and $\Gamma_{N}$ with $\bar{\Gamma}_{D} \cap \bar{\Gamma}_{N}=\emptyset$. (This because we want to treat simultaneously both Dirichlet and Neumann conditions without bothering about the compatibility conditions.)

We assume that $f \in L^{2}(D), g \in H^{1}(D)$ and $u_{0} \in H^{2}(D)$. Then it is well known that the problem (2.1) has a unique solution $u \in H^{2}(\Omega), u-u_{0} \in H^{1}\left(\Omega ; \Gamma_{D}\right)$.

We shall study the dependence of $u$ on the shape of $\Omega$. For that we construct a family of domains $\Omega_{t}$ as follows. Let us choose a vector field $V \in C^{1}\left(D ; R^{n}\right)$ and introduce a deformation map $T_{t}: x \rightarrow x+t V(x)$ which is injective for small $t$. We denote $\Omega^{t}=T_{t}(\Omega)=\{x+t V(x) \mid x \in \Omega\}$.

In $\Omega^{t}$ we define the stated problem as follows:

$$
\begin{aligned}
-\Delta u_{t} & =f, & & \text { in } \Omega^{t}, \\
u_{t} & =u_{0}, & & \text { on } \Gamma_{D}^{t}, \\
\frac{\partial u_{t}}{\partial n} & =g, & & \text { on } \Gamma_{N}^{t} .
\end{aligned}
$$

We address the question of continuous dependence using the concept of a shape derivative. We define the shape derivative of $u$ to direction $V$ as the limit

$$
u_{V}^{\prime}=\left.\lim _{t \rightarrow 0+} \frac{\tilde{u}_{t}-u}{t}\right|_{\Omega},
$$

where ? denotes any regularity preserving extension from $\Omega^{t}$ to $D$. The limit does not depend on the choice of the extension.

Theorem 2.1. Under the above assumptions there exists a shape derivative $u_{V}^{\prime} \in$ $H^{1}(\Omega)$. Moreover, $u_{V}^{\prime}$ is the unique solution of the problem

$$
\begin{aligned}
-\Delta u_{V}^{\prime} & =0, \quad \text { in } \Omega, \\
u_{V}^{\prime} & =-\frac{\partial\left(u-u_{0}\right)}{\partial n}\langle V, n\rangle, \quad \text { on } \Gamma_{D}, \\
\frac{\partial u_{V}^{\prime}}{\partial n} & =-\nabla_{\Gamma} \cdot\left(\langle V, n\rangle \nabla_{\Gamma} u\right)+\left(f+H g+\frac{\partial g}{\partial n}\right)\langle V, n\rangle, \quad \text { on } \Gamma_{N} .
\end{aligned}
$$

Here $H$ stands for the mean curvature of $\Gamma$ and $\nabla_{\Gamma}$ denotes the tangential part of $\nabla$, that is, $\nabla_{\Gamma} u=\nabla u-(\partial u / \partial n) n$.

The proof of this theorem can be obtained by combining the proofs of Propositions 3.1 and 3.3 of [9]. This result is almost what we need to analyze the error due to polyhedral approximation of the geometry. Namely, let $\Omega$ be a $C^{2}$ domain and $\Omega_{h}$ its polyhedral approximation so that the vertices of $\partial \Omega_{h}$ are on $\partial \Omega$. Then, if $h$ is small enough we can locally describe $\partial \Omega$ and $\partial \Omega_{h}$ with the same charts and deduce from standard interpolation theory that $\left\|\Omega-\Omega_{h}\right\|_{C^{0}} \leq C h^{2}$, $\left\|\Omega-\Omega_{h}\right\|_{C^{0,1}} \leq C h$, where $C$ depends on the $C^{2}$-smoothness of $\partial \Omega$. Hence we can write $\partial \Omega_{h}=\left\{x+h V_{h}(x) \mid x \in \partial \Omega\right\}$, where $\left\|V_{h}\right\|_{L^{\infty}} \leq C h,\left\|V_{h}\right\|_{W^{1, \infty}} \leq C$. Clearly, problem (2.7) is well defined for such $V_{h}$. Moreover, we can estimate the norm of $u^{\prime}$ as

$$
\left\|u^{\prime}\right\|_{1, \Omega} \leq C\left(\left\|u^{\prime}\right\|_{1 / 2, \Gamma_{D}}+\left\|\frac{\partial u^{\prime}}{\partial n}\right\|_{-1 / 2, \Gamma_{N}}\right)
$$


Now,

$$
\begin{aligned}
\left\|u^{\prime}\right\|_{1 / 2, \Gamma_{D}} & \leq\left\|-\frac{\partial\left(u-u_{0}\right)}{\partial n}\langle V, n\rangle\right\|_{0, \Gamma_{D}}^{1 / 2}\left\|-\frac{\partial\left(u-u_{0}\right)}{\partial n}\langle V, n\rangle\right\|_{1, \Gamma_{D}}^{1 / 2} \\
& \leq\left\|-\frac{\partial\left(u-u_{0}\right)}{\partial n}\right\|_{1, \Gamma_{D}}\|\langle V, n\rangle\|_{L^{\infty}}^{1 / 2}\|\langle V, n\rangle\|_{W^{1, \infty}}^{1 / 2} \\
& \leq C h^{1 / 2}
\end{aligned}
$$

provided that $u$ and $u_{0}$ belong to $H^{5 / 2}(\Omega)$. Similarly,

$$
\left\|\frac{\partial u^{\prime}}{\partial n}\right\|_{-1 / 2, \Gamma_{N}} \leq\left\|\langle V, n\rangle \nabla_{\Gamma} u\right\|_{1 / 2, \Gamma_{N}}+\left\|\left(f+H g+\frac{\partial g}{\partial n}\right)\langle V, n\rangle\right\|_{L^{2}, \Gamma_{N}} \leq C h^{1 / 2}
$$

if $u \in H^{5 / 2}(\Omega), f \in H^{1 / 2}(\Omega)$ and $g \in H^{3 / 2}(\Omega)$.

This suggests the estimate $\left\|u_{\Omega_{h}}-u_{\Omega}\right\|_{1} \leq C h^{3 / 2}$ in the case of sufficiently smooth data. However, the above development is only formal as the velocity field $V$ does not satisfy the conditions of the shape differentiability proof. Also the estimate $\left\|u^{\prime}\right\|_{1} \leq C h^{3 / 2}$ does not imply automatically the $h^{3 / 2}$ estimate for the difference of the two solutions as the differentiability proof of 9] does not give a quantitative estimate for

$$
\left\|\frac{1}{t}\left(\tilde{u}_{t}-u\right)-u^{\prime}\right\|_{1}
$$

Theorem 2.2. Let $V \in W^{1, \infty}(D)$. Then if $u$ and $u_{0}$ belong to $H^{5 / 2}, f \in H^{1}(\Omega)$, and $g \in H^{1}(\Omega)$, and $\tilde{u}$ is an $H^{5 / 2}$ extension of $u$ to $D$, it holds that

$$
\left\|u_{t}-\tilde{u}\right\|_{1, \Omega^{t}} \leq C t\|V\|_{L^{\infty}}^{1 / 2}\|V\|_{W^{1, \infty}}^{1 / 2} .
$$

Proof. We start by writing the variational problems for $u_{t}$ and $u$ in $\Omega^{t}$. For $u_{t}$ we have directly

$$
\int_{\Omega^{t}} \nabla u_{t} \nabla \phi=\int_{\Omega^{t}} f \phi+\int_{\Gamma_{N}^{t}} g \phi \quad \forall \phi \in H^{1}\left(\Omega^{t} ; \Gamma_{D}^{t}\right)
$$

and $u_{t}-u_{0} \in H^{1}\left(\Omega^{t} ; \Gamma_{D}^{t}\right)$. For $u$ we have

$$
\int_{\Omega} \nabla u \nabla \phi=\int_{\Omega} f \phi+\int_{\Gamma_{N}} g \phi \quad \forall \phi \in H^{1}\left(\Omega ; \Gamma_{D}\right)
$$

with $u-u_{0} \in H^{1}\left(\Omega ; \Gamma_{D}\right)$. Now let us denote $u^{t}=u \circ T_{t}^{-1} \in H^{1}\left(\Omega^{t}\right)$. It is easy to see that if we choose $\phi=\hat{\phi} \circ T_{t}, \hat{\phi} \in H^{1}\left(\Omega^{t} ; \Gamma_{D}^{t}\right)$ in (2.10), we can rewrite

$$
\begin{aligned}
\int_{\Omega} \nabla u \nabla \phi & =\int_{\Omega^{t}} D T_{t} \nabla u^{t} D T_{t} \nabla \hat{\phi}\left|D T_{t}^{-1}\right|, \\
\int_{\Omega} f \phi & =\int_{\Omega^{t}} f \circ T_{t}^{-1} \hat{\phi}\left|D T_{t}^{-1}\right|, \\
\int_{\Gamma_{N}} g \phi & =\int_{\Gamma_{N}^{t}} g \circ T_{t}^{-1} \hat{\phi} s_{t},
\end{aligned}
$$

where $s_{t}=\left|D T_{t}\right|\left\|D T_{t}^{-\top} n\right\|$ (see [9]).

We are now ready to write an equation for $\delta u=u_{t}-\tilde{u}$ :

$$
\int_{\Omega^{t}} \nabla \delta u \nabla \phi=\left\langle R_{t}, \phi\right\rangle \quad \forall \phi \in H^{1}\left(\Omega^{t} ; \Gamma_{D}^{t}\right)
$$


with $\delta u-\left(\tilde{u}-u_{0}\right) \in H^{1}\left(\Omega^{t} ; \Gamma_{D}^{t}\right)$ and

$$
\begin{aligned}
\left\langle R_{t}, \phi\right\rangle & =\int_{\Omega^{t}}\left(\nabla \tilde{u} \nabla \phi-D T_{t} \nabla u^{t} D T_{t} \nabla \phi\left|D T_{t}^{-1}\right|-f \phi+f \circ T_{t}^{-1} \phi\left|D T_{t}^{-1}\right|\right) \\
& -\int_{\Gamma_{N}^{t}} g \phi-g \circ T_{t}^{-1} \phi s_{t} .
\end{aligned}
$$

Now if we can show that the data of the problem 2.11) is sufficiently small, we obtain the conclusion as the problem 2.11) is invertible uniformly with respect to $\Omega^{t}$. We formulate the estimates for the data in the following two lemmas.

Lemma 2.3. If $u_{0}, \tilde{u} \in H^{5 / 2}(D)$, then

$$
\left\|u_{0}-\tilde{u}\right\|_{1 / 2, \Gamma_{D}^{t}} \leq C t\|V\|_{L^{\infty}}^{1 / 2}\|V\|_{W^{1, \infty}}^{1 / 2} .
$$

Lemma 2.4. If $\tilde{u} \in H^{5 / 2}(D), f \in H^{1}(D)$ and $g \in H^{1}(D)$, then

$$
\left\|R_{t}\right\|_{-1, \Omega^{t}} \leq C t\|V\|_{L^{\infty}}^{1 / 2}\|V\|_{W^{1, \infty}}^{1 / 2} .
$$

For the proofs of Lemmas 2.3 and 2.4 we shall need some results on the differentiability with respect to deformation $T_{t}$.

Lemma 2.5. Let $f \in W^{1, p}(D)$ for some $p<\infty$ and $V \in W^{1, \infty}$. Then

$$
\left\|\frac{1}{t}\left(f \circ T_{t}-f\right)-\nabla f \cdot V\right\|_{L^{p}} \rightarrow 0
$$

as $t \rightarrow 0$.

Proof. First let $f \in C^{\infty}$. Then for any $x \in \Omega$

$$
\frac{1}{t}\left(f \circ T_{t}-f\right)(x)-\nabla f(x) \cdot V(x)=\int_{0}^{1}(\nabla f(x+s t V(x)) d s-\nabla f(x)) \cdot V(x) .
$$

Hence

$$
\begin{aligned}
\sup _{\|\psi\|_{L^{p^{\prime}}}=1} & \left(\frac{1}{t}\left(f \circ T_{t}-f\right)-\nabla f \cdot V, \psi\right) \\
\leq & \sup _{\|\psi\|_{L^{p^{\prime}}=1}} \int_{0}^{1} \int_{\Omega} \psi(\nabla f(x+s t V(x))-\nabla f(x)) \cdot V(x) d x d s \\
\leq & \int_{0}^{1}\left\|\left(\nabla f \circ T_{s t}-\nabla f\right) \cdot V\right\|_{L^{p}} .
\end{aligned}
$$

For any $s, \nabla f \circ T_{s t}$ is continuous with respect to $t$ in $L^{p}$. Hence the above integral tends to zero. By the density argument we can extend the result for $f \in W^{1, p}$.

With similar arguments we can also prove

Lemma 2.6. Let $f \in W^{2, p}(D)$ for some $p<\infty$ and $V \in W^{1, \infty}$. Then

$$
\left\|\frac{1}{t}\left(f \circ T_{t}-f\right)-\nabla f \cdot V\right\|_{W^{1, p}} \rightarrow 0
$$

as $t \rightarrow 0$.

Obviously, analogous results hold when $T_{t}$ is replaced by $T_{t}^{-1}$.

Now we are ready to prove Lemma 2.3. 
Proof. As $T_{t}$ maps $H^{1}(\Omega)$ functions to $H^{1}\left(\Omega^{t}\right)$ functions, it also maps $H^{1 / 2}\left(\Gamma_{D}\right)$ to $H^{1 / 2}\left(\Gamma_{D}^{t}\right)$. Hence

$$
\begin{aligned}
\left\|u_{0}-\tilde{u}\right\|_{1 / 2, \Gamma_{D}^{t}} & \leq C\left\|\left(u_{0}-\tilde{u}\right) \circ T_{t}^{-1}\right\|_{1 / 2, \Gamma_{D}} \\
& =C\left\|\left(u_{0}-\tilde{u}\right) \circ T_{t}^{-1}-\left(u_{0}-\tilde{u}\right)\right\|_{1 / 2, \Gamma_{D}} \\
& \leq C\left\|t\left(\nabla\left(u_{0}-\tilde{u}\right)+\xi_{t}\right) \cdot V\right\|_{1 / 2, \Gamma_{D}}
\end{aligned}
$$

for some $\left\|\xi_{t}\right\|_{1, \Omega} \rightarrow 0$ as we get from Lemma 2.6 that $\left(\left(u_{0}-\tilde{u}\right) \circ T_{t}^{-1}-\left(u_{0}-\tilde{u}\right)\right) / t$ converges to $-\nabla\left(u_{0}-\tilde{u}\right) \cdot V$ in $H^{1}(\Omega)$ and, consequently, also in $H^{1 / 2}\left(\Gamma_{D}\right)$.

So the leading term of $\left\|u_{0}-\tilde{u}\right\|_{1 / 2, \Gamma_{D}^{t}}$ can be estimated as

$$
\left\|\frac{\partial\left(u_{0}-u\right)}{\partial n} t\langle V, n\rangle\right\|_{1 / 2, \Gamma_{D}} \leq\left\|\frac{\partial\left(u_{0}-u\right)}{\partial n} t\langle V, n\rangle\right\|_{L^{2}\left(\Gamma_{D}\right)}^{1 / 2}\left\|\frac{\partial\left(u_{0}-u\right)}{\partial n} t\langle V, n\rangle\right\|_{1, \Gamma_{D}}^{1 / 2}
$$

from which the conclusion follows.

Next we shall prove Lemma 2.4.

Proof. We start from the boundary term $\int_{\Gamma_{N}^{t}} g \phi-g \circ T_{t}^{-1} \phi s_{t}$. First we observe that $\left|s_{t}\right|=1-t \nabla_{\Gamma} \cdot V+O\left(t^{2}\|V\|_{W^{1, \infty}}^{2}\right)$. From Lemma 2.5 we get that $\left(g-g \circ T_{t}^{-1}\right) / t$ converges to $\nabla g \cdot V$ in $L^{2}(D)$ with a remainder term of order $o\left(\|V\|_{L^{\infty}}\right)$. Thus for any $\phi \in H^{1}\left(\Omega^{t}\right)$ we can write

$$
\int_{\Gamma_{N}^{t}} g \phi-g \circ T_{t}^{-1} \phi s_{t}=t \int_{\Gamma_{N}^{t}}\left(\nabla g \cdot V+g \nabla_{\Gamma} \cdot V\right) \phi+O\left(t^{2}\|V\|_{W^{1, \infty}}^{2}\right)+o\left(t\|V\|_{L^{\infty}}\right) .
$$

Integrating by parts on $\Gamma_{N}^{t}$, ([9], Chapter 2.23), we get the leading term into the form

$$
\int_{\Gamma_{N}^{t}}\left(\frac{\partial g}{\partial n}+g H\right)\langle V, n\rangle \phi
$$

Next let us consider the term involving $f$. As $f \in H^{1}(D)$ we can deduce from Lemma 2.5 that $f-f \circ T_{t}^{-1}=-t \nabla f \cdot V-t \xi_{t} \cdot V$ for some $\xi_{t} \rightarrow 0$ strongly in $L^{2}(D)^{n}$. Hence,

$$
\begin{aligned}
\int_{\Omega^{t}} f \phi-f \circ T_{t}^{-1} \phi\left|D T_{t}^{-1}\right| & \approx-t \int_{\Omega^{t}}(\nabla f \cdot V+\nabla \cdot V f) \phi \\
& =-t\left(\int_{\Omega^{t}}-f V \cdot \nabla \phi+\int_{\partial \Omega^{t}} f \phi\langle V, n\rangle\right) \\
& =-t\left(\int_{\Omega^{t}}-\Delta u_{t} V \cdot \nabla \phi+\int_{\Gamma_{N}^{t}} f \phi\langle V, n\rangle\right) .
\end{aligned}
$$

Finally, we have to reformulate also the terms involving $u$. With Lemma 2.6 we get that $u \circ T_{t}^{-1} \approx \tilde{u}-t V \cdot \nabla \tilde{u}$ with smaller terms tending to zero fast enough in $H^{1}$. Consequently we can write

$$
\begin{aligned}
\int_{\Omega^{t}} \nabla \tilde{u} \nabla \phi-D T_{t} \nabla u^{t} D T_{t} \nabla \phi\left|D T_{t}^{-1}\right| & \approx \int_{\Omega^{t}} \nabla(t V \cdot \nabla \tilde{u}) \nabla \phi \\
& -t\left(D V+D V^{\top}-I \nabla \cdot V\right) \nabla \tilde{u} \nabla \phi .
\end{aligned}
$$


Now integrating by parts twice we have

$$
\begin{aligned}
\int_{\Omega^{t}} \nabla(V \cdot \nabla \tilde{u}) \nabla \phi & =\int_{\Omega^{t}} D V \nabla \tilde{u} \nabla \phi+\int_{\Omega^{t}} D^{2} \tilde{u} V \nabla \phi \\
=\int_{\Omega^{t}}(D V-I \nabla \cdot V) \nabla \tilde{u} \nabla \phi & -\int_{\Omega^{t}} D^{2} \phi \nabla \tilde{u} V+\int_{\partial \Omega^{t}} \nabla \tilde{u} \nabla \phi\langle V, n\rangle \\
=\int_{\Omega^{t}}\left(D V+D V^{\top}-I \nabla \cdot V\right) \nabla \tilde{u} \nabla \phi & +\int_{\Omega^{t}} \Delta \tilde{u} V \cdot \nabla \phi \\
& +\int_{\partial \Omega^{t}} \nabla \tilde{u} \nabla \phi\langle V, n\rangle-\frac{\partial \tilde{u}}{\partial n} \nabla \phi \cdot V .
\end{aligned}
$$

Combining the above derivations we conclude that the leading part of $R_{t}$ can be written as

$$
\left\langle R_{t}, \phi\right\rangle=t \int_{\Gamma_{N}^{t}}\left(\left(f+g H+\frac{\partial g}{\partial n}\right) \phi+\nabla_{\Gamma} \tilde{u} \nabla_{\Gamma} \phi\right)\langle V, n\rangle .
$$

From the proofs of Lemmas 2.3 and 2.4 one can observe that the leading terms in the data for $\delta u$ can in fact be identified with the data characterizing the shape derivative $u_{V}^{\prime}$.

In the above results the regularity requirements for data are not in balance. Partly this is due to the fact that we showed convergence in $L^{2}$ for the $f$ term instead of an $H^{-1}$-estimate that would be sufficient. Let us note, that for $f$ in $L^{2}(D)$ one cannot prove strong differentiability under $T_{t}$ in $H^{-1}$-norm (see [9]). Whether this could be done for $f \in H^{s}(D)$ for some $s<1$ is not known. Also in the treatment of the essential boundary condition, no effort has been made to optimize the regularity (last estimate in the proof of Lemma 2.3).

If we now apply the above result to a smooth domain and its polyhedral approximation, we obtain an $O\left(h^{3 / 2}\right)$ estimate for the energy norm of the error in the solution. Similar estimates have been obtained already by Strang and Berger [10] and Thomée [11] in the early 1970's under the assumption that the domain to be approximated was convex.

For the purpose of finite element error estimates in the energy norm, an $O(h)$ estimate is sufficient. It can be obtained quite easily with weaker assumptions on the regularity of the data.

Theorem 2.7. Let $\tilde{u}, u_{0} \in H^{2}(D), f \in L^{2}(D)$ and $g \in H^{1}(D)$. Then

$$
\left\|u_{t}-\tilde{u}\right\|_{1, \Omega^{t}} \leq C t\|V\|_{W^{1, \infty}} .
$$

The above estimate can be obtained by writing

$$
\left\|u_{t}-\tilde{u}\right\|_{1, \Omega^{t}} \leq\left\|u_{t}-u \circ T_{t}^{-1}\right\|_{1, \Omega^{t}}+\left\|u \circ T_{t}^{-1}-\tilde{u}\right\|_{1, \Omega^{t}} .
$$

It is easy to write an equation for the first term and to bound its data. The second term can be bounded using Lemma 2.6. Observe that $u \circ T_{t}^{-1}$ can be considered to be an "extension" of $u$ to $\Omega^{t}$ in the sense of Section 1 .

It is also of interest to derive estimates in the $L^{\infty}$ norm. For the Dirichlet problem some estimates follow as easy consequences of the previous developments. For example, 
Theorem 2.8. Assume that $\partial \Omega=\Gamma_{D}, V \in W^{1, \infty}(D), u, u_{0} \in W^{2, p}, f \in W^{1, p}$ for some $p>n$ and that $\tilde{u}$ is a $W^{2, p}$ extension of $u$ to $D$. Then it holds that

$$
\left\|u_{0}-\tilde{u}\right\|_{L^{\infty}\left(\Gamma_{D}^{t}\right)} \leq C t\|V\|_{L^{\infty}} .
$$

Proof. Thanks to the Sobolev imbedding of $W^{1, p}(D)$ to $L^{\infty}(D)$, we can observe from the proof of Lemma2.3 that $\left\|u_{0}-\tilde{u}\right\|_{L^{\infty}\left(\Gamma_{D}^{t}\right)} \leq C t\left\|\partial\left(u_{0}-\tilde{u}\right) / \partial n\langle V, n\rangle\right\|_{L^{\infty}\left(\Gamma_{D}^{t}\right)} \leq$ $C t\|V\|_{L^{\infty}}$.

While this estimate is of optimal order, the regularity assumptions are not sharp. Better estimates for the Dirichlet case have been obtained by Thomée [11], who also provides examples showing the sharpness of the $O\left(h^{2}\right)$-estimate with respect to both $L^{\infty}$ and $L^{2}$ norms. In the Neumann case we are not aware of any $L^{\infty}$-estimates.

We conclude this section by discussing the finite element error estimates related to above geometric estimates. In the case of the estimate in the energy norm, the situation is very simple. Thanks to Poincare's inequality and Cea's lemma we obtain easily that the quasi-optimality result holds with uniform constant. Hence the developments of the previous section lead immediately to

Theorem 2.9. Let $\Omega_{h}$ be a polygonal approximation of $\Omega$. Define the discrete problem as standard piecewise linear finite element approximation of (2.4) for $t=h$. Then for the error between the discrete solution $u_{h}$ and the prolongated solution $\tilde{u}$, it holds that

$$
\left\|\tilde{u}-u_{h}\right\|_{H^{1}\left(\Omega_{h}\right)} \leq C h\|f\|_{L^{2}(\Omega)} .
$$

For other norms the situation is not so easy. For example, for $L^{\infty}$-estimates an appealing starting point could be the quasi-optimality result by Rannacher and Scott [6] which states that

$$
\left\|\hat{u}-u_{h}\right\|_{1, p, \Omega_{h}} \leq C \inf _{w_{h} \in V_{h}}\left\|\hat{u}-w_{h}\right\|_{1, p, \Omega_{h}} \quad 2 \leq p \leq \infty .
$$

However, the question of whether the constant $C$ can be chosen independently of $\Omega_{h}$ was not addressed in 6]. On the other hand there exist some uniform quasioptimality results like that of Schatz and Wahlbin [8] which state that under several technical conditions for the discrete domains one has the estimate

$$
\left\|u-u_{h}\right\|_{L^{\infty}} \leq C X(h) \inf _{w_{h} \in V_{h}}\left\|w_{h}-u\right\|_{L^{\infty}}
$$

where $X(h)=1$ except in the two dimensional case where it equals to $-\ln h$. To generalize this result to the case where $u$ is replaced by $\hat{u}$ that depends on $\Omega_{h}$ may be even harder than to prove the result directly.

\section{SECOND ORDER SYSTEMS}

Let us next consider the case where the state problem is a system like the elasticity problem or the (Navier) Stokes equations. It turns out that the question of continuous dependence on geometry is more delicate in this case. To illustrate this, we shall consider a model problem in elasticity with several different boundary conditions.

Let us write the problem in an abstract setting as

$$
a(u, \phi)=F(\phi) \quad \forall \phi \in W .
$$

Here

$$
W=\left\{\phi \in\left(H^{1}(\Omega)\right)^{d} \mid \phi=0 \text { on } \Gamma_{0}, \phi \cdot n=0 \text { on } \Gamma_{1}\right\} .
$$


The bilinear form $a$ is defined as

$$
a(u, \phi)=\int_{\Omega} \sigma_{i j}(u) \epsilon_{i j}(\phi),
$$

where

$$
\epsilon(\phi)=\frac{1}{2}\left(D \phi+D^{\top} \phi\right)
$$

and the stress tensor $\sigma$ is defined as

$$
\sigma_{i j}(u)=C_{i j k l} \epsilon_{k l}(u) .
$$

In this and next section we use the standard convention of summation over repeated indices. The fourth order tensor of elastic coefficients, $C$, satisfies the standard symmetry conditions

$$
C_{i j k l}=C_{j i k l}=C_{k l i j}
$$

and the coercivity condition

$$
C_{i j k l} \psi_{i j} \psi_{k l} \geq c \psi_{i j} \psi_{i j}
$$

for some $c>0$ and for all symmetric second order tensors $\psi$. Finally the data $F$ is given by

$$
F(\phi)=\int_{\Omega} f_{i} \phi_{i}+\int_{\Gamma_{2}} g_{i} \phi_{i}
$$

for some $f \in\left(L^{2}(D)\right)^{d}, g \in\left(L^{2}(D)\right)^{d}$. The boundary of $\Omega$ is decomposed as $\partial \Omega=\bar{\Gamma}_{0} \cup \bar{\Gamma}_{1} \cup \bar{\Gamma}_{2}$.

If $\left|\Gamma_{0}\right|_{d-1}>0$, the problem (3.1) is coercive and, consequently, its solvability is obvious.

Let us now address the question of continuous dependence on geometry. As in the previous section, we start by stating a shape differentiability result. First we formulate sufficient conditions for shape differentiability:

- The velocity field $V \in C^{2}(D)$ with $V=0$ on $\bar{\Gamma}_{0} \cap \bar{\Gamma}_{1}, \bar{\Gamma}_{0} \cap \bar{\Gamma}_{2}, \bar{\Gamma}_{1} \cap \bar{\Gamma}_{2}$.

- $f \in\left(C^{1}(D)\right)^{d}, g \in\left(C^{1}(D)\right)^{d}, C \in\left(C^{1}(D)\right)^{d^{4}}$.

- $D u \cdot V \in\left(H^{1}(\Omega)\right)^{d}$.

Under these conditions the solution of (3.1) is shape differentiable to direction $V$ and the shape derivative $u^{\prime}$ solves the problem

$$
\begin{aligned}
\nabla \cdot \sigma & =0, \quad \text { in } \Omega, \\
u^{\prime} & =-\langle V, n\rangle \frac{\partial u}{\partial n}, \quad \text { on } \Gamma_{0}, \\
u^{\prime} \cdot n & =u_{\tau} \cdot\left(D V^{\top} \cdot n\right), \quad \text { on } \Gamma_{1}, \\
\sigma\left(u^{\prime}\right)_{\tau} & =\langle V, n\rangle f_{\tau}, \quad \text { on } \Gamma_{1}, \\
\sigma\left(u^{\prime}\right) \cdot n & =\langle V, n\rangle(f+H g)-\nabla_{\Gamma} \cdot\left(\langle V, n\rangle \sigma_{\tau}\right), \quad \text { on } \Gamma_{2} .
\end{aligned}
$$

Here $\sigma_{\tau}=\sigma \cdot n-(\sigma \cdot n \cdot n) n$ and $u_{\tau}, f_{\tau}$ denote the tangential components of $u$ and $f$, respectively. For the proof, see [9, Theorem 3.11].

If $\Gamma_{1}=\emptyset$, we can apply the techniques of the previous section to extend the above results for $W^{1, \infty}$ velocity fields as well. 
Theorem 3.1. Let $\Gamma_{1}=\emptyset, V \in W^{1, \infty}(D), V=0$ on $\bar{\Gamma}_{0} \cap \bar{\Gamma}_{2}$. Then if $u$ and $u_{0}$ belong to $H^{5 / 2}, f \in H^{1}(\Omega)$ and $g \in H^{1}(\Omega)$, and $\tilde{u}$ is an $H^{5 / 2}$ extension of $u$ to $D$, it holds that

$$
\left\|u_{t}-\tilde{u}\right\|_{1, \Omega^{t}} \leq C t\|V\|_{L^{\infty}}^{1 / 2}\|V\|_{W^{1, \infty}}^{1 / 2} .
$$

Proof. As in the proof of Theorem 2.2, we can show that the difference $u_{t}-\tilde{u}$ solves a problem with data whose leading terms coincide with the data of the problem characterizing the shape derivative $u^{\prime}$. Hence we can conclude that

$$
\left\|u_{t}-\tilde{u}\right\|_{1 / 2, \Gamma_{0}^{t}} \leq\left\|\langle V, n\rangle \frac{\partial u}{\partial n}\right\|_{1 / 2, \Gamma_{0}^{t}} \leq C t\|V\|_{L^{\infty}}^{1 / 2}\|V\|_{W^{1, \infty}}^{1 / 2}
$$

and

$$
\left\|\sigma\left(u_{t}-\tilde{u}\right) \cdot n\right\|_{-1 / 2, \Gamma_{2}^{t}} \leq\left\|\langle V, n\rangle(f+H g)-\nabla_{\Gamma} \cdot\left(\langle V, n\rangle \sigma_{\tau}\right)\right\|_{-1 / 2, \Gamma_{2}^{t}} .
$$

If $\Gamma_{1}$ is present, we cannot obtain shape differentiability in $H^{1}$ for $W^{1, \infty}$ velocity fields. This follows directly from the fact that

$$
u^{\prime}=u_{\tau} \cdot\left(D V^{\top} \cdot n\right)
$$

does not belong to $H^{1 / 2}\left(\Gamma_{1}\right)$ anymore if $V$ has only $W^{1, \infty}$ regularity. In fact, it turns out that the solution does not change continuously under polygonal perturbation of the boundary (see [2, 14]).

This result implies that the finite element approximation of this problem has to be made more carefully than just by approximating the domain by a polyhedric one. If polyhedric approximation is used, the "locking" it implies has to be treated by relaxing the boundary condition. This can be done for example by "underintegration" (i.e., imposing the Dirichlet condition only at discrete points, 14]) or by introducing a regularized normal field. The latter approach can be treated in our abstract framework. Namely, let us assume that we can define a smoothing operator $P$ such that the discrete problem satisfies the boundary condition $u_{h} \cdot P\left(n_{\Omega_{h}}\right)=0$. Then, the "geometric" part of the approximation error contains the error due to changing $n$ to $P(n)$ in the original problem and the error due to approximating $\Omega$ with $\Omega_{h}$ for the problem with the regularized boundary condition $u \cdot P(n)=0$.

In the two dimensional case such a smoothing operator can be obtained as a convolution with a one dimensional hat function of length-scale $h$. As typical estimates for such convolutions are of the type $\|P(\phi)\|_{s} \leq C / h\|\phi\|_{s-1}$, only suboptimal error estimates can be expected. This is in accordance with [14] as well. Stabilization techniques needed for optimal convergence rates (see 15]) seem to be inherently discrete and cannot be treated with our technique.

\section{A FOURTh ORDER MODEL PROBLEM}

In this section we consider the continuous dependence on geometry for higher order problems. As a model example we take the linear Kirchhoff plate. Thus, let $w \in H^{4}(\Omega)$ be the transversal deflection of the plate $\Omega \subset R^{2}$, which satisfies the equation

$$
\left(b_{i j k l} w_{, k l}\right)_{, i j}=f \quad \text { in } \Omega
$$


with the boundary conditions

$$
\begin{aligned}
& w=0, \quad \frac{\partial w}{\partial n}=0 \quad \text { on } \Gamma_{0}, \\
& w=0, \quad M_{n}=0 \quad \text { on } \Gamma_{1}, \\
& M_{n}=0, \quad Q=0 \quad \text { on } \Gamma_{2},
\end{aligned}
$$

where $\partial \Omega=\bar{\Gamma}_{0} \cup \bar{\Gamma}_{1} \cup \bar{\Gamma}_{2}$,

$$
M_{n}=M_{i j} n_{i} n_{j}
$$

with $M_{i j}=b_{i j k l} w_{, k l}$ denotes the moment, and

$$
Q=-M_{k l, l} n_{k}-\frac{\partial}{\partial \tau}\left(M_{k l} n_{l} \tau_{k}\right)
$$

is the shear force. The coefficient tensor $b \in R^{2^{4}}$ satisfies the standard symmetry and coercivity conditions.

The corresponding weak form can be written as

$$
a(w, \phi)=F(\phi) \quad \forall \phi \in W, w \in W,
$$

where

$$
W=\left\{\phi \in H^{2}(\Omega) \mid \phi=0 \text { on } \Gamma_{0} \cup \Gamma_{1}, \frac{\partial \phi}{\partial n}=0 \text { on } \Gamma_{0}\right\}
$$

and

$$
\begin{aligned}
a(w, \phi) & =\int_{\Omega} b_{i j k l} w_{, i j} \phi_{, k l}, \\
F(\phi) & =\int_{\Omega} f \phi .
\end{aligned}
$$

If $\Gamma_{0} \neq \emptyset$, the solvability is guaranteed for all $f \in W^{\prime}$.

Next we state, without proof, a shape differentiability result from [9]: If the data $(\Omega, b, f)$, the deformation velocity $V$, and the solution $w$ are smooth enough, then the solution $w$ is shape differentiable to direction $V$ and the shape derivative $w^{\prime}$ is the unique solution of the problem

$$
\begin{array}{rlrl}
\left(b_{i j k l} w_{, k l}^{\prime}\right)_{, i j} & =0 \quad \text { in } \Omega, \\
w^{\prime} & =0, \quad \frac{\partial w^{\prime}}{\partial n}=\langle V, n\rangle \frac{\partial^{2} w}{\partial n^{2}} \quad \text { on } \Gamma_{0}, & \\
w^{\prime} & =-\langle V, n\rangle \frac{\partial w}{\partial n} \quad \text { on } \Gamma_{1}, & \\
M_{n}^{\prime} & =\nabla_{\Gamma}\left(\langle V, n\rangle M_{n \tau}\right)+\nabla_{\Gamma}\left(\langle V, n\rangle M_{\tau}\right) \cdot n & & \text { on } \Gamma_{1} \cup \Gamma_{2}, \\
Q^{\prime} & =-\nabla_{\Gamma} \cdot\left(\nabla_{\Gamma} \cdot\left(\langle V, n\rangle M_{\tau}\right)_{\tau}\right)+\langle V, n\rangle f & & \text { on } \Gamma_{2},
\end{array}
$$

where

$$
\begin{aligned}
M_{n}^{\prime} & =b_{i j k l} w_{, k l}^{\prime} n_{i} n_{j}, \\
\left(M_{\tau}\right)_{i j} & =M_{i j}-M_{n} n_{i} n_{j} .
\end{aligned}
$$

As before we shall use the equation for the shape derivative to get error estimates for the solution under perturbation of the geometry. First we notice that conforming approximation of fourth order problems requires the use of $C^{1}$-elements. Hence, if we use similar approximation for the geometry as well, we are lead to study deformation velocities in $W^{2, \infty}$. In this case, proceeding as in the case of the Laplace equation, we can show that the leading term in the difference between $w_{t}$, 
the solution in the perturbed domain, and $\tilde{w}$, the $H^{2}$-extension of $w$ to $\Omega_{t}$, is $t w^{\prime}$. Hence, to estimate the error due to a change of domain it is sufficient to get a bound for $w^{\prime}$.

Now, to get an estimate for $w^{\prime}$ in $H^{2}(\Omega)$, we have to bound $w^{\prime}$ in $H^{3 / 2}\left(\Gamma_{0} \cup \Gamma_{1}\right)$, $\frac{\partial w^{\prime}}{\partial n}$ in $H^{1 / 2}\left(\Gamma_{0}\right), M_{n}^{\prime}$ in $H^{-1 / 2}\left(\Gamma_{1} \cup \Gamma_{2}\right)$ and $Q^{\prime}$ in $H^{-3 / 2}\left(\Gamma_{2}\right)$.

For $w^{\prime}$ we have

$$
\left\|w^{\prime}\right\|_{3 / 2, \Gamma_{1}} \leq\left\|w^{\prime}\right\|_{1, \Gamma_{1}}^{1 / 2}\left\|w^{\prime}\right\|_{2, \Gamma_{1}}^{1 / 2} \leq C\|V\|_{1, \infty}^{1 / 2}\|V\|_{2, \infty}^{1 / 2}\left\|\frac{\partial w}{\partial n}\right\|_{2, \Gamma_{1}} .
$$

Similarly, for the other terms

$$
\begin{gathered}
\left\|\frac{\partial w^{\prime}}{\partial n}\right\|_{1 / 2, \Gamma_{1}} \leq C\|V\|_{L^{\infty}}^{1 / 2}\|V\|_{1, \infty}^{1 / 2}\left\|\frac{\partial^{2} w}{\partial n^{2}}\right\|_{1, \Gamma_{1}}, \\
\left\|M_{n}^{\prime}\right\|_{-1 / 2, \Gamma_{1} \cup \Gamma_{2}} \leq C\|V\|_{L^{\infty}}^{1 / 2}\|V\|_{1, \infty}^{1 / 2}\|M\|_{1, \Gamma_{1} \cup \Gamma_{2}}
\end{gathered}
$$

and

$$
\left\|Q^{\prime}\right\|_{-3 / 2, \Gamma_{2}} \leq C\|V\|_{L^{\infty}}^{1 / 2}\|V\|_{1, \infty}^{1 / 2}\|M\|_{1, \Gamma_{2}}+C\|V\|_{L^{\infty}}\|f\|_{-1, \Gamma_{2}} .
$$

Thus summarizing the above estimates we get

$$
\begin{aligned}
\left\|w^{\prime}\right\|_{2, \Omega} \leq & C\|V\|_{1, \infty, \Gamma_{1}}^{1 / 2}\|V\|_{2, \infty, \Gamma_{1}}^{1 / 2}\|w\|_{7 / 2, \Omega}+C\|V\|_{L^{\infty}}^{1 / 2}\|V\|_{1, \infty}^{1 / 2}\|w\|_{7 / 2, \Omega} \\
& +C\|V\|_{L^{\infty}}\|f\|_{-1 / 2, \Omega} .
\end{aligned}
$$

This estimate cannot be transformed directly to an error estimate as such since some higher order terms with respect to $V$ require somewhat higher regularity from the data (at least $f \in L^{2}(\Omega)$ ).

Now if $\Omega_{h}$ is an approximation of $\Omega$ resulting from a $C^{1}$-cubic spline approximation of the boundary $\partial \Omega$ and if $\Omega$ is a $C^{4}$-surface, we have that $\left|\Omega-\Omega_{h}\right|=O\left(h^{4}\right)$ and that $\Omega_{h}$ can be obtained as the image of a $W^{2, \infty}$ deformation map $T_{h}=I+h V$, where $\|V\|_{2-k, \infty} \leq C h^{1+k}, k=0,1,2$. This results further to the estimate

$$
\left\|w_{h}-\tilde{w}\right\|_{2, \Omega} \approx h\left\|w^{\prime}\right\|_{2, \Omega} \leq C h^{5 / 2}
$$

in the general case and to

$$
\left\|w_{h}-\tilde{w}\right\|_{2, \Omega} \leq C h^{7 / 2}
$$

in the case where $\Gamma_{1}=\emptyset$. One observes thus that the solution is more sensitive to the approximation of $\Gamma_{1}$ than to approximation of other boundaries. In both cases, however, the geometric error is asymptotically smaller than the $O\left(h^{2}\right)$ error related to finite element approximation by $C^{1}$-elements.

In the case of polyhedral approximation of $\Omega$ one observes a situation analogous to the elasticity case. For $V \in W^{1, \infty}$, the problem for $w^{\prime}$ admits a solution only if $\Gamma_{1}=\emptyset$. However, unlike in the case of second order problems we cannot deduce continuous dependence on geometry from this result as the proof of shape differentiability and hence the derivation of the corresponding problem requires higher derivatives of the deformation velocity. In the case where $\Gamma_{1}$ is present, the solution is not continuous with respect to polyhedral approximation of the geometry. This is demonstrated in the classical counterexample of [1], the so-called Babuska's plate paradox. 


\section{Conclusions}

We have analyzed an error due to polyhedral approximation of smooth boundaries in the continuous setting. The analysis shows that for scalar second order problems the geometric part of the approximation error can be neglected at least if the energy norm is considered. Thus for those problems not much can be gained by using other than isoparametric fitting of the elements at the boundary. The technique used for the error analysis is independent of the finite element method used (Galerkin, Petrov-Galerkin, mixed, etc.) provided the method can be shown uniformly quasi-optimal in a family of polyhedral domains. The technique can be applied also to more complicated cases such as coupling of integral equations with boundary value problems. For this, see $[12$.

For systems and higher order equations the situation is more interesting. For some boundary conditions the analysis can be performed as in the scalar case while for some other cases the analysis fails, indicating a lack of continuous dependence under polyhedral approximation of the geometry. Thus formal shape analysis can be used as a quick way to identify the boundary conditions with which one should be especially careful when treating curved boundaries.

\section{ACKNOWLEDGMENTS}

This work is an outgrowth of a presentation given at Jyväskylä in July 1997, [13. The feedback from a.o. M. Kř́ǐzek, R. Stenberg and L. Wahlbin is gratefully

acknowledged. The main part of this work has been supported by the Academy of Finland.

\section{REFERENCES}

[1] Babuska I., Stabilität des Definitionsgebietes mit Rücksicht auf grundlegende Probleme der Theorie des partiellen Differentialgleichungen auch in Zusammenhang mit der Elasticitätstheorie, Czechoslovak Math. J. 11, 1961, pp. 76-105, 165-203. MR 23:A2629

[2] Babuska I., The theory of small changes in the domain of existence in the theory of partial differential equations and its applications, in Differential equations and their applications, Academic Press, New York, 1963, pp. 13-26. MR 53:7070

[3] Berger A., Scott R., Strang G., Approximate boundary conditions in the finite element method, in Symposia Mathematica, Vol X, Academic Press, London, 1972, pp. 295-313. MR 53:7070

[4] Ciarlet P.G., Raviart P.A., Interpolation theory over curved elements with applications to finite element methods, Comp. Meth. Appl. Mech. Eng., 1, 1972, pp. 217-249. MR 51:11991

[5] Lenoir M., Optimal isoparametric finite elements and error estimates for domains involving curved boundaries, SIAM J. Num. Anal., 1986, pp. 562-580. MR 87m:65163

[6] Rannacher R., Scott R., Some optimal error estimates for piecewise linear finite element approximations, Math. Comp. 38, 1982, pp. 437-445. MR 83e:65180

[7] Scott R. Interpolated boundary conditions in the finite element method, SIAM J. Num. Anal., 12, 1975, pp. 404-427. MR 52:7162

[8] Schatz A.H., Wahlbin L.B., On the quasi-optimality in $L^{\infty}$ of the $H_{0}^{1}$-projection into finite element spaces, Math. Comp. 38, 1982, pp. 1-22. MR 82m:65106

[9] Sokolowski J., Zolésio J.P., Introduction to shape optimization, shape sensitivity analysis, Springer, Berlin, 1992. MR 94d:49002

[10] Strang G., Berger A. E., The change in solution due to change in domain, Proceedings of symposia in pure mathematics, XXIII, Ed. D.C. Spencer, AMS, vol. 23, 1973, pp. 199-205. MR 49:1796

[11] Thomée V., Polygonal domain approximation in Dirichlet's problem, Journal of IMA, 11, 1973, pp. 33-44. MR 50:1538 
[12] Tiihonen T., Finite element approximation of nonlocal heat radiation problems, Math. Mod. Meth. Appl. Sci., 8, 1998, pp. 1071-1089. MR 99h:65172

[13] Tiihonen T., Shape calculus and FEM in smooth domains, in Finite Element Methods: Superconvergence, Post-processing and A Posteriori Estimates, eds. Kř́̌žek M., Neittanmäki P., Stenberg, R., Marcel Dekker, New York, 1997, pp. 259-267. MR 99c:49041

[14] Verfürth R., Mixed finite element approximation of a fluid flow problem, in The mathematics of finite elements and applications V, ed. J. Whiteman, Academic Press, 1985, pp. 335-342. MR 87a:65184

[15] Verfürth R., Finite element approximation of incompressible Navier-Stokes equations with slip boundary condition. II, Num. Math. 59, 1991, pp. 616-636. MR 92k:65170

[16] Zlamal M., Curved elements in the finite element method, I, II, SIAM J. Num. Anal., 10, 1973, pp. 229-240, 11, 1974, pp. 347-362. MR 52:16060; MR 49:8400

Department of Mathematical Information Technology, University of Jyväskylä, Box 35, FIN-40351 JyvÄSKYlä, FinLAND

E-mail address: tiihonen@it.jyu.fi 